\title{
Numerical investigation of aerodynamic noise generated by circular cylinders in cross-flow at Reynolds numbers in the upper subcritical and critical regimes
}

\author{
Xiaowan Liu ${ }^{1,2}$, David J Thompson ${ }^{1}$, Zhiwei Hu ${ }^{2}$
}

\begin{abstract}
A numerical investigation of the aeroacoustic characteristics of the flow past a circular cylinder is presented for Reynolds numbers in the range $2.67 \times 10^{4}-3.67 \times 10^{5}$, which falls within the upper subcritical and critical regimes. This is based on Computational Fluid Dynamics simulations using a Delayed Detached-Eddy Simulation (DDES) model for the aerodynamics of the near-field, which feeds the equivalent source terms into the Ffowcs Williams-Hawkings equation for far-field noise prediction. The accuracy of DDES in predicting unsteady flow quantities is assessed from an engineering viewpoint through comparisons with experimental data. Good agreement is found for both the near-field flow quantities and the far-field noise spectra. The aerodynamic and aeroacoustic characteristics are investigated from two aspects, the effect of varying the Reynolds number and the sensitivity to the spanwise computational dimension. The results in terms of the vortex shedding frequency, hydrodynamic forces and far-field noise levels only show small variations in the subcritical range. However in the critical range, the vortex shedding frequency increases and the noise level decreases considerably after allowing for the typical sixth power dependence. A spanwise length of $3 D$ is found to be sufficient for most Reynolds numbers in the critical range, but in the subcritical range, a longer spanwise length is needed and the sound level may be under-predicted by up to around $4.5 \mathrm{~dB}$ by using $3 D$.
\end{abstract}

\section{Keywords}

circular cylinder, aerodynamic noise, CFD, Aeolian tone, pantograph

\section{Introduction}

The aerodynamic noise due to flow over cylinders has been studied extensively as it can represent wide range of applications in many engineering fields. Flow over a cylinder typically causes Aeolian tones, which were first found by Strouhal and have been shown to be associated with the vortex shedding phenomenon. The particular interest of the present work is the noise radiated by the components of a train pantograph, which can be modelled as circular cylinders. The pantograph is one of the most important aerodynamic noise sources on a high speed train since it cannot be shielded by conventional noise barriers (Thompson et al. 2015). There are many other applications, notably aircraft landing gear.

The factors affecting the far-field pressure radiated by a rigid circular cylinder can be illustrated from the following equation for the mean-square pressure due to vortex shedding Fujita (2010),

$$
\overline{p^{2}(r)}=\frac{\rho_{0}^{2} U_{\infty}^{6} S t_{p}^{2} C_{L, \mathrm{rms}}^{2} L l_{c}}{16 c_{0}^{2} r^{2}}
$$

where $\rho_{0}$ is the air density, $c_{0}$ is the sound speed and $r$ is the distance between the noise source and the observer. The cylinder has a span length $L$ and diameter $D, U_{\infty}$ is the freestream velocity and $S t_{p}$ is the normalized frequency, or Strouhal number $\left(=f D / U_{\infty}\right)$ at the peak, where $f$ is the vortex shedding frequency. $l_{c}$ is the spanwise correlation length (usually normalized by $D, l_{c}=l_{c D} D$ ), and $C_{L, \mathrm{rms}}$ is the root mean square (rms) of the lift coefficient $C_{L}$, which is defined by $C_{L}=\frac{F_{\text {lift }}}{0.5 \rho U_{\infty}^{2} D L}$ with $F_{\text {lift }}$ the lift force. Equation (1) is derived from Curle's equation (Curle 1955) and has been used by Fujita (2010) and King and Pfizenmaier (2009) among others for the prediction of noise from cylinders. This equation neglects convective effects. It is valid when the compact source condition and the far-field condition are satisfied. A compact source means that the characteristic length of the noise source is much smaller than the radiated acoustic wavelength. For Aeolian tone radiation, which is of interest in the current study, the characteristic length is considered to be the correlation length of the pressure fluctuations on the cylinder surface. The far-field condition means that the distance between the noise source and the observer is much larger than the acoustic wavelength.

In Equation (1), the parameters $S t_{p}, C_{L, \text { rms }}$ and $l_{c}$ depend on the Reynolds number ( $R e=U_{\infty} D / \nu$, where $\nu$ is the kinematic viscosity). The variation of these parameters is caused by the flow conditions, especially the location

${ }^{1}$ Institute of Sound and Vibration Research, University of Southampton, Southampton, SO17 1BJ, UK

${ }^{2}$ Aerodynamic and Flight Mechanics Research Group, University of Southampton, SO17 1BJ, UK

\section{Corresponding author:}

Xiaowan Liu, Institute of Sound and Vibration Research, University of Southampton, Southampton, SO17 1BJ, UK.

Email: xl12y17@soton.ac.uk 
of the transition where flow changes from laminar to turbulent. Zdravkovich (1997) defined a number of flow regimes classified by the Reynolds number according to the location of the transition. Of particular interest to the current pantograph case are Reynolds numbers in the range, $R e=2 \times 10^{4}-4 \times 10^{5}$, which fall in the upper subcritical and critical flow states. The onset of the critical flow state, marked by a pronounced decrease in the drag coefficient $C_{D}$ (where $C_{D}=\frac{F_{\mathrm{drag}}}{0.5 \rho U_{\infty}^{2} D L}$ ), mostly happens in the range $R e=1 \times 10^{5}-3 \times 10^{5}$. With different experimental conditions, such as different surface roughness and inflow turbulence levels, the value of critical Reynolds number can vary significantly (Wieselsberger 1922; Fage and Warsap 1930; Delany and Sorensen 1953; Bearman 1969; Schewe 1983; Farell and Blessmann 1983). For example, Norberg and Sunden (1987) have shown that this critical Reynolds number is shifted from $2 \times 10^{5}$ to $1 \times 10^{5}$ when the inflow turbulence level is increased from $0.1 \%$ and $1.4 \%$. Other experimental setups, such as wind tunnel blockage ratio, ratio of cylinder length to diameter and end conditions, also influence this Reynolds number.

For the subcritical flow state, the boundary layer remains laminar until separation and the transition to turbulence occurs in the separated shear layers (Zdravkovich 1997). The values of $C_{D}, S t_{p}$ and $C_{L, \text { rms }}$ are found to be more or less insensitive to $R e$ with $C_{D} \approx 1.2$ (Wieselsberger 1922; Fage and Warsap 1930; Roshko 1961; Cantwell and Coles 1983), and $S t_{p} \approx 0.2$ (Delany and Sorensen 1953; Bearman 1969; Cantwell and Coles 1983). Breuer (2000) and Kim (2006) performed Large Eddy Simulations (LES) for a Reynolds number of $1.4 \times 10^{5}$ and obtained good agreement with measurements for $C_{D}$ and $S t_{p}$. The capabilities of the Detached Eddy Simulation (DES) approach for revealing the significant flow features at $R e=1.4 \times 10^{5}$ were investigated by Travin et al. (2000) who obtained an over-predicted vortex formation length, and therefore an under-predicted drag coefficient. These discrepancies with experiments are very likely due to the dissipative upwind integration scheme adopted in their method. Mockett et al. (2009) compared their DES results, using a low-dissipative hybrid scheme of upwind and central differencing, with Particle Image Velocimetry measurement data and showed commendable agreement for both timeaveraged and unsteady quantities.

When increasing the Reynolds number to the critical range, the transition to turbulence happens immediately after the flow separation, which can disturb the separation and delay the eddy formation. This leads to a sharp decrease in $C_{D}$ and $C_{L, \text { rms }}$, and an increase in $S t_{p}$. Bearman (1969) observed two major drops in $C_{D}$ with the first drop accompanied by an intermediate asymmetric flow state in which a laminar separation bubble consistently forms on one side of the cylinder only. This asymmetric flow state was earlier observed by Achenbach (1968) who suggested that it might be caused by the test conditions since the flow in this regime is rather sensitive to any small disturbances. When the second drop happens, the flow recovers symmetry with the separation bubble appearing on both sides. Regarding the vortex shedding, a large scatter in $S t_{p}$ exists in the measurements. Bearman (1969) reported a value of 0.32 when one bubble forms and 0.46 for two bubbles. These results are consistent with the measured values of Schewe (1983). Irregular shedding processes, characterised by broad peaks in the spectrum of $C_{L}$, were also found within this range just before the appearance of the bubble (Schewe 1983). The sensitivity of the flow to the location of the transition to turbulence makes this regime very challenging for both experimental and numerical investigations. Only limited numerical studies exist in this regime. Among these, Roshko (1961) analysed the flow topology by using LES and Cheng et al. (2017) provided an insight from a skin friction perspective through LES. Commendable agreement with the measurements has been obtained in these LES studies with respect to the variations in $C_{D}$ and $S t_{p}$.

The spanwise correlation is also one of the main factors affecting vortex-induced sound generation. The knowledge of the spanwise correlation is particularly important in determining a sufficient spanwise computational dimension in three-dimensional numerical simulations to capture the dynamic features of the largest flow structures. The spanwise correlation coefficients are not well studied in the literature. In the measurements of West and Apelt (1997) for $R e=$ $2.2 \times 10^{4}-1.3 \times 10^{5}$, the correlation coefficients were found to reduce to below 0.2 for a spanwise spacing of $8 D$, and the variations with $R e$ were insignificant. Norberg (2003) summarized a trend of $l_{c} / D$ versus $R e$ based on a collection of measured data, which showed that the spanwise correlation length decreases from about $4 D$ to $3.4 D$ when $R e$ is increased from $3 \times 10^{4}$ to $1 \times 10^{5}$. Most numerical simulations have used spanwise computational dimensions of between $1 D$ and $\pi D$ (Breuer 2000; Kim 2006; Travin et al. 2000; Mockett et al. 2009). Breuer (2000) performed simulations with two spanwise domain lengths, $2 D$ and $\pi D$, and found only small changes in $C_{D}$ and $S t_{p}$. Travin et al. (2000) demonstrated that a length of $2 D$ can provide fair agreement with the experimental results of Cantwell and Coles (1983). However, through numerical investigations of spanwise correlation coefficients at $R e=1.66 \times 10^{5}$, Lockard et al. (2007) pointed out that only a span of $18 D$ was able to capture the complete decay in the correlation.

Regarding the noise radiated by circular cylinders, most existing studies are for the Reynolds numbers below the critical range. King and Pfizenmaier (2009) conducted experimental investigations for $R e=4.4 \times 10^{4}-1.4 \times$ $10^{5}$. As one free end was used in their measurements, it was found that the length-to-diameter ratio should be greater than 25 to avoid its influence on the noise results. An experimental study on vortex shedding noise characteristics of circular cylinders can also be found in Latorre Iglesias et al. (2016), who measured the peak Strouhal number, noise level and directivity for Reynolds numbers up to $1.2 \times 10^{5}$.

The information in the current literature on radiated noise is rather limited for the critical Reynolds number range. Fujita (2010) measured the Aeolian tone and surface fluctuations along the cylinder span for $R e=2.5 \times 10^{5}-$ $2 \times 10^{6}$. A significant increase in $S t_{p}$ was found when $R e \geq 3.2 \times 10^{5}$, which was considered to be the beginning of the critical flow regime. In addition, they demonstrated much weakened spanwise correlations in the critical regime, together with a dramatic reduction of about $18 \mathrm{~dB}$ in the peak levels of the Aeolian tone. 
The sensitivity of the flow in the critical regime has been widely pointed out in the literature. This increases the difficulties in both experiments and numerical simulations, and thus a large scatter appears in the results. LES simulations of Rodríguez et al. (2015) and Cheng et al. (2017) have shown the capabilities of representing the main flow features in this regime. However, the LES approach is too computationally costly to be used for engineering applications in the present case. In spite of limitations of DES, this approach has been shown to be practical tool that can provide reasonable agreement at subcritical Reynolds numbers (Travin et al. 2000; Mockett et al. 2009). Rather than attempting a detailed investigation of physical mechanisms, which demands an approach that is accurate enough to capture the transition to turbulence, the current study aims to explore the feasibility of using DES for noise prediction of cylinder flow in the upper subcritical and critical regimes. Furthermore, as spanwise correlation is important for noise prediction but only limited information exists, the current study will investigate the spanwise correlation for the relevant subcritical and critical Reynolds numbers, analyse the influence of the spanwise computational dimension, and provide an approximate correction for noise results when a short spanwise dimension is used.

The numerical methodology applied in this work is introduced in Section 2 which also describes the computational setup. A grid sensitivity study is presented in Section 3. Results on the effect of the Reynolds number are given in Section 4, where results are compared with measurement data and analysed in terms of both flow and noise characteristics. The effect of the spanwise computational dimension on the sound level is assessed in Section 5.

\section{Numerical method}

\section{Computational methodology}

Aerodynamic sound is generated by unsteady fluid motion, which is governed by the continuity and momentum equations, as given below, together with the energy equation.

$$
\begin{aligned}
\frac{\partial \rho}{\partial t}+\frac{\partial \rho u_{i}}{\partial x_{i}} & =0 \\
\frac{\partial \rho u_{i}}{\partial t}+\frac{\partial}{\partial x_{j}}\left(\rho u_{i} u_{j}+p \delta_{i j}-\tau_{i j}\right) & =0
\end{aligned}
$$

where $\rho$ is the density, $\tau_{i j}$ is the viscous stress tensor, $p$ is the pressure, $u_{i}$ is the flow velocity component in the $x_{i}$ direction. Here, the tensor summation convention is used, and $i, j=1 \sim 3$ for the three coordinate directions.

Numerical methods need to be adopted to obtain solutions to the Navier-Stokes equations. For the purpose of industrial application, the Reynolds-Averaged Navier-Stokes (RANS) method is often preferred, but it cannot predict flow unsteadiness. LES can be used to resolve the large, energycontaining eddies, but it requires significant computational resources, especially for wall-bounded flow where very fine cells are required. Therefore, hybrid unsteady RANS-LES methods were developed to provide a remedy for both the limited capability of RANS simulations and the relatively high computational cost of LES simulations. Specifically, the RANS model is applied in the boundary layer to save computational cost whereas the LES model is enabled away from the boundary layer to resolve the large scale unsteadiness.

The most common hybrid RANS-LES method is DES, which was first proposed by Spalart (2000) who applied the Spalart-Allmaras (S-A) turbulence model for the RANS region. The switching between RANS and LES is based on a characteristic length scale,

$$
\tilde{d}=\min \left\{d ; C_{D E S} \Delta\right\}
$$

where $d$ is the distance to the wall, $\Delta$ is a length scale specified by $\Delta=\max \left\{\Delta_{x} ; \Delta_{y} ; \Delta_{z}\right\}$ (where $\Delta_{x}, \Delta_{y}, \Delta_{z}$ are grid sizes in the $x-, y-, z$-direction, respectively) and the constant $C_{D E S}=0.65$ is calibrated for isotropic turbulence. When close to the wall, $d<C_{D E S} \Delta$, the RANS model is employed. Further away from the wall $(d>$ $\left.C_{D E S} \Delta\right)$, the LES mode is enabled. Although the DES model has proved successful in simulating certain flows, it still has some drawbacks. The criterion for the switch from RANS to LES can lead to Modelled Stress Depletion (Fröhlich and von Terzi 2008). This happens when the grid size is small enough to activate the LES mode inside the boundary layer but not fine enough to support accurate LES content. In such a situation, the eddy viscosity, and therefore the modelled Reynolds stress, drops below the RANS level, but the mesh is not fine enough for LES to create adequately resolved stresses to restore the balance. This normally occurs when a user gradually refines the grid to seek grid convergence. This drawback can lead to unphysical outcomes, such as Grid Induced Separation. In order to avoid this limitation, a Delayed DES (DDES) model was proposed by Spalart et al. (2006) which is an improved version of the DES model. For DDES, a function $f_{d}$ was added to define the length scale,

$$
\tilde{d}=d-f_{d} \max \left\{0 ; d-C_{D E S} \Delta\right\}
$$

where

$$
f_{d}=1-\tanh \left[\left(8 r_{d}\right)^{3}\right] \quad \text { and } \quad r_{d}=\frac{\nu_{t}+\nu}{\sqrt{u_{i, j} u_{i, j}} k^{2} d^{2}}
$$

in which $\nu$ is the kinematic viscosity, $\nu_{t}$ is the turbulent eddy viscosity, $u_{i, j}$ is the velocity gradient component for $u_{i}$ in the $j^{\text {th }}$ direction, $k$ is the von Karman constant, and $d$ is the distance to the wall. The value of $r_{d}$ equals 1 inside the boundary layer, and falls gradually to 0 towards the edge of the boundary layer. With the introduction of the delaying function $f_{d}$, it is ensured that the attached boundary layer is solved in RANS mode.

After obtaining the unsteady flow from CFD simulations, the flow information can be used as input to calculate the far-field noise from the following Ffowcs Williams and Hawkings (FW-H) equation (Williams and Hawkings 1969).

$$
\begin{aligned}
\frac{\partial^{2} \bar{\rho}^{\prime}}{\partial t^{2}}-c_{0}^{2} \nabla^{2} \bar{\rho}^{\prime}=\frac{\partial}{\partial x_{i} \partial x_{j}} & {\left[T_{i j} H(f)\right] } \\
& -\frac{\partial}{\partial x_{i}}\left[F_{i} \delta(f)\right]+\frac{\partial}{\partial t}[Q \delta(f)]
\end{aligned}
$$


where $\delta(f)$ is the Dirac delta function, and $H(f)$ is the Heaviside function. $H(f)=0$ inside the integral surface $(f<0)$ and $H(f)=1$ elsewhere $(f \geq 0) \cdot \bar{\rho}^{\prime}$ is a 'windowed' variable, which is given by $\rho(\mathbf{x}, t)$ outside the surface and is constant and equal to $\rho_{0}$ inside the surface,

On the right hand side of Equation (7), the first term, $T_{i j}=\rho u_{i} u_{j}+\left(p-c_{0}^{2} \rho^{\prime}\right)-\tau_{i j}$, is the quadrupole source term which normally results from non-linear aerodynamic phenomena such as non-linear wave propagation, shocks, vorticity and turbulence in the flow field. The second term, $F_{i}=P n_{i}-\tau_{i j} n_{j}+\rho u_{i}\left(u_{n}-v_{n}\right)$, is the dipole source term which is generated by the unsteady forces acting on the fluid from the object. The third term, $Q=\rho_{0} v_{n}+\rho\left(u_{n}-\right.$ $\left.v_{n}\right)$, is the monopole source term which represents the noise generated by unsteady mass flux through the surface. $u_{n}$ is the fluid velocity component normal to the FW-H integral surface defined by $f=0, v_{n}$ is the normal velocity of the surface.

In the present study, since the interest is in low subsonic flow $(M<0.3)$, the influence of compressibility is small, and the Navier-Stokes equations are solved in incompressible form. The contribution of the quadrupole sources is not considered since it is much less significant compared with other contributions for these low Mach numbers. The FW$\mathrm{H}$ integral surface is chosen as the cylinder surface. Lockard et al. (2007) have used the FW-H equation to predict noise radiated from circular cylinders, and found that there were only minor differences between the results of using onsurface and off-surface integration surfaces for a Mach number of 0.166 . When the FW-H is evaluated over the solid surface and $v_{n}=0$ the monopole term also vanishes. Therefore only the dipole source due to wall pressure fluctuations is considered.

\section{Computational set-up}

Computational domain and boundary conditions The cylinder modelled has a diameter $D$ of $0.05 \mathrm{~m}$. As shown in Figure 1(a), the computational domain extends over $31 D$ in the $x$-direction, with the centre of the cylinder located at $10.5 \mathrm{D}$ from the upstream boundary. In the $y$-direction, the width is $21 D$ with an equal distance of $10.5 D$ from the cylinder centre to the top and bottom. In the spanwise direction, a length of $3 D$ was chosen according to the literature (Breuer 2000; Kim 2006; Travin et al. 2000; Mockett et al. 2009). The extent to which the noise results will be influenced by a short spanwise dimension will be discussed in Section 5 where more simulations with longer spanwise domain length will be considered.

The upstream boundary $(x=-10.5 D)$ is set to the velocity inlet condition and the downstream boundary $(x=$ $+20.5 D)$ is set to the pressure outlet condition. The solid surface of the cylinder is set to a non-slip wall. The top and bottom boundaries are set to symmetry conditions. To simulate cylinders with an infinite span, periodic boundary conditions are imposed in the spanwise direction. Different levels of turbulent viscosity ratio between 1 and 5 for the $\mathrm{S}$-A model were tested at the inlet and little difference was found in the results. This is because the influence of the inflow boundary condition diminishes rapidly, and the viscosity ratio has little influence on flow near the cylinder. The value of this ratio has been examined in the simulation
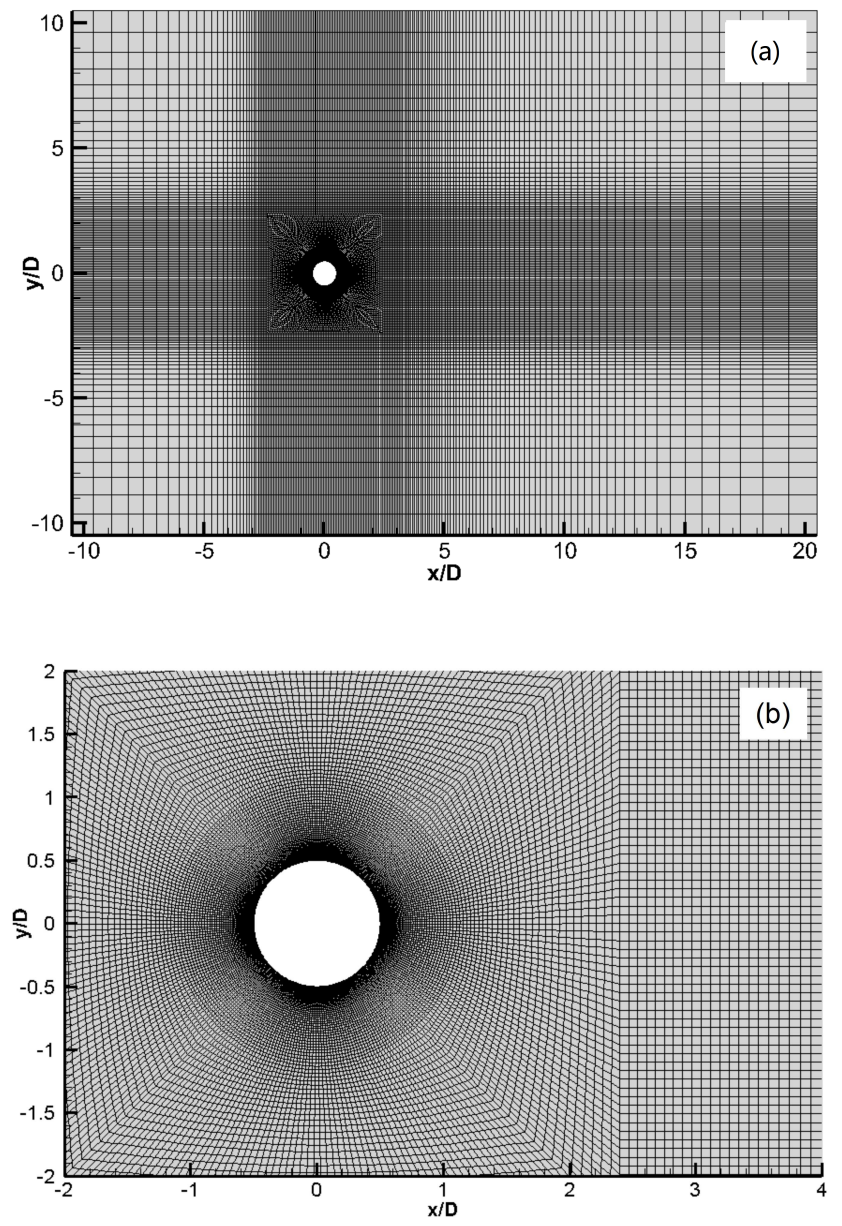

Figure 1. Grid configuration. (a) whole domain, (b) in the vicinity of the cylinder.

results and found to be very low in the boundary layer until flow separation. Travin et al. (2000) observed a turbulent separation, not found in the current simulations, by setting inflow and initial values of 5 for this ratio. The reason for this inconsistency is unclear.

The simulated cases for various inflow speeds, corresponding to different Reynolds numbers, are listed in Table 1 , along with the number of cells in the $x-y$ plane and along the spanwise direction. The number of cells around the cylinder surface $\left(N_{s}\right)$ is also given. This is chosen based on the principles obtained from a grid sensitivity study that will be discussed later in Section 3. This table also contains the non-dimensional time step $\Delta t U_{\infty} / D$ used for the simulations. The number of cells increases to maintain the mesh quality for higher Reynolds numbers, e.g. the same maximum aspect ratios (which appears in the first layer cell adjacent to the cylinder surface). In this work, the S-A model is adopted in DDES for flow within the boundary layer, with a very fine wall-normal spacing near the wall, $y_{1}^{+} \leq 1$. $y_{1}^{+}$is a non-dimensional wall distance, $y_{1}^{+}=u_{\tau} y_{1} / \nu$ where $u_{\tau}$ is the friction velocity at wall, $\nu$ is the kinematic viscosity of the fluid and $y_{1}$ is the height of the first layer cell adjacent to the cylinder surface. A structured mesh is generated for all the cases. The grids are stretched with a growth ratio of 1.05 near the cylinder and 1.1 further away. The grids are refined up to $5 D$ downstream of the cylinder centre to 
Table 1. Computational parameters

\begin{tabular}{ccccc}
\hline$U_{\infty}[\mathrm{m} / \mathrm{s}]$ & $R e$ & Grid & $N_{s}$ & $\Delta t U_{\infty} / D$ \\
\hline \hline 8 & $2.67 \times 10^{4}$ & $4119 \times 42$ & 46 & 0.008 \\
15 & $5 \times 10^{4}$ & $7412 \times 66$ & 75 & 0.0075 \\
22 & $7.33 \times 10^{4}$ & $11359 \times 86$ & 98 & 0.006 \\
30 & $1 \times 10^{5}$ & $14586 \times 115$ & 124 & 0.009 \\
35 & $1.17 \times 10^{5}$ & $14586 \times 115$ & 124 & 0.0085 \\
45 & $1.5 \times 10^{5}$ & $17575 \times 135$ & 140 & 0.009 \\
60 & $2 \times 10^{5}$ & $26623 \times 165$ & 170 & 0.0095 \\
110 & $3.67 \times 10^{5}$ & $58905 \times 291$ & 320 & 0.005 \\
\hline
\end{tabular}

provide a good resolution of the unsteady flow in the wake, as shown in Figure 1. The unsteady flow simulation is carried out in OpenFOAM 2.3.0, while the aerodynamic noise is predicted in FLUENT with input of the flow information on the cylinder surface after obtaining the surface pressure fluctuations .

\section{Grid sensitivity study}

To ensure the validity of the calculations, the effects on turbulence statistics of the spatial resolution, the temporal resolution and different numerical schemes for the convection terms have been investigated for $R e=1 \times$ $10^{5}\left(U_{\infty}=30 \mathrm{~m} / \mathrm{s}\right)$. At this Reynolds number, laminar separation happens on the verge of transition which makes it more grid-sensitive compared with turbulent separation cases (Travin et al. 2000). Travin et al. (2000) and Breuer (2000) have found that mesh convergence is difficult to achieve at Reynolds numbers in the upper subcritical regime. In addition, the switch to the LES mode, which is activated after massive separations in the present simulations, increases this difficulty as the filter width depends on the grid resolution. Therefore, in the present study, grid changes are viewed more as tests of soundness and sensitivity than as a search for convergence.

It is not realistic to perform the grid sensitivity study at all Reynolds numbers as the present study involves simulations of flow at 8 speeds, and for the highest Reynolds number, the grid contains nearly 20 million cells; using 240 processors, it still needs at least 4 weeks to finish one simulation. Considering the computational cost and the large amount of work, the Reynolds number chosen as the reference case to check the grid sensitivity was located in the middle of the range considered, at the upper end of the subcritical Reynolds number range. The meshes generated for other Reynolds numbers (see Table 1) follow the guidelines obtained after the grid sensitivity study in this section.

Table 2 lists all the cases run for the grid sensitivity study. $A R_{s}$ and $A R_{z}$ represent the aspect ratios in the first boundary layer cell of the circumferential and spanwise size to the wall-normal size respectively. These are also the maximum aspect ratios for the whole domain since $\Delta y$ here is $y_{1}$ which is the smallest grid size in the wallnormal direction. The dimensionless time step $\left(\Delta t U_{\infty} / D\right)$ of 0.009 corresponds to a physical time step of $1.5 \times$ $10^{-5} \mathrm{~s}$, and ensures that the maximum Courant-FriedrichsLewy (CFL) number is less than 2. The CFL number is defined as: $C F L=\Delta t U_{\infty} / \Delta x$, where $\Delta x$ is the smallest cell size in the streamwise direction. Although a second-order implicit scheme is used for the time integration, which can provide stable solutions for much higher CFL numbers, an appropriate time step should still be determined to resolve the physics of the flow and retain the computational efficiency. The pressure-velocity coupling term is modelled by the PIMPLE algorithm, which combines the SIMPLE and PISO algorithms (Versteeg and Malalasekera 2007).

The influences of two discrezation schemes, second-order upwind ( $2^{\text {nd }}$ order UP) and Limited Linear, are investigated for the convection term in Case 1 and Case 2. Cases 3 and 4 check the temporal resolution and the spanwise grid resolution respectively on the basis of Case 2. In Cases 2, 5 and 6, both spatial and temporal resolutions are considered. The grid was refined by a factor of $\sqrt{2}$ in both $x-$ and $z-$ directions and the time step was reduced to maintain the same maximum CFL. This refinement strategy is the same as considered by Travin et al. (2000) where the grid is refined in all directions simultaneously, instead of one at a time. Case 7 includes a further refinement of the wake region on the basis of Case 5. Based on Case 7, the temporal resolution and the spanwise grid resolution are examined in Cases 8 and 9 , respectively.

Results are reported in Table 3 in terms of the rms lift coefficient, $C_{L, \text { rms }}$, mean drag coefficient $C_{D}$, peak Strouhal number $S t_{p}$, and base pressure coefficient $C_{p b}$ (pressure coefficient at the back of the cylinder). The pressure coefficient, $C_{p}$, is defined as, $C_{p}=\frac{p-p_{\infty}}{0.5 \rho U_{\infty}^{2}}$ with $p$ the pressure on the cylinder surfaces, and $p_{\infty}$ the reference pressure chosen as the pressure at the inlet. $\theta_{\text {sep }}$ is the separation angle, estimated based on where the skin friction crosses zero. $L_{r}$ is the recirculation length from the back of the cylinder to the zero-mean-velocity point on the centreline, and is normalized by $D$. Experimental results from Cantwell and Coles (1983), LES results from Breuer (2000) and DES results from Travin et al. (2000) all at $R e=$ $1.5 \times 10^{5}$ are given for comparison. In Breuer's LES results, Case B1 is the refined mesh based on Case A1, but the results from Case $\mathrm{A} 1$ give a better agreement with experiments.

Temporal convergence is achieved after an initial time duration of $0.2 \mathrm{~s}$ as the values of $C_{L, \mathrm{rms}}$ and $C_{D}$ do not change with longer sampling time. The final statistics were gathered from $0.25 \mathrm{~s}$ to $1.0 \mathrm{~s}$, corresponding to 450 nondimensional time units $\left(t^{*}=t U_{\infty} / D\right)$, which is sufficient to obtain a representative sample of 90 shedding cycles (for $S t_{p}=0.2$ ). Table 3 shows a strong correlation among $L_{r},-C_{p b}, C_{D}$ and $C_{L, \text { rms. }}$. A shortened recirculation length $L_{r}$ corresponds to higher $-C_{p b}, C_{D}$ and $C_{L, \text { rms. }}$. This is consistent with the findings of Travin et al. (2000). 
Table 2. Numerical resolutions and schemes for grid sensitivity study

\begin{tabular}{cccccc}
\hline Cases & Grid & $A R_{s}$ & $A R_{z}$ & $\Delta t U_{\infty} / D$ & Scheme \\
\hline \hline 1 & $10926 \times 81$ & 175 & 200 & 0.009 & $2^{\text {nd }}$ order UP \\
2 & $10926 \times 81$ & 175 & 200 & 0.009 & Limited linear \\
3 & $10926 \times 81$ & 175 & 200 & 0.0045 & Limited linear \\
4 & $10926 \times 151$ & 175 & 100 & 0.009 & Limited linear \\
5 & $13050 \times 115$ & 135 & 140 & 0.009 & Limited linear \\
6 & $16950 \times 151$ & 100 & 100 & 0.0045 & Limited linear \\
7 & $14586 \times 115$ & 135 & 140 & 0.009 & Limited linear \\
8 & $14586 \times 115$ & 135 & 140 & 0.0045 & Limited linear \\
9 & $14586 \times 151$ & 135 & 100 & 0.009 & Limited linear \\
\hline
\end{tabular}

Table 3. Summary of the global flow parameters from the grid sensitivity study

\begin{tabular}{ccccccc}
\hline Cases & $C_{D}$ & $C_{l, \text { rms }}$ & $S t_{p}$ & $\theta_{\text {sep }}\left(^{\circ}\right)$ & $-C_{p b}$ & $L_{r} / D$ \\
\hline \hline 1 & 0.91 & 0.25 & 0.214 & 81 & 0.72 & 1.1 \\
2 & 1.07 & 0.43 & 0.2 & 82 & 0.82 & 0.9 \\
3 & 1 & 0.37 & 0.2 & 82 & 0.78 & 0.95 \\
4 & 1.17 & 0.68 & 0.183 & 85 & 0.98 & 0.72 \\
5 & 1.19 & 0.65 & 0.19 & 83 & 1 & 0.78 \\
6 & 1.35 & 1.12 & 0.183 & 90 & 1.25 & 0.4 \\
7 & 1.25 & 0.73 & 0.19 & 84 & 1.1 & 0.6 \\
8 & 1.18 & 0.68 & 0.19 & 84 & 0.9 & 0.75 \\
9 & 1.25 & 0.75 & 0.19 & 87 & 1 & 0.5 \\
Exp Cantwell and Coles (1983) & 1.24 & - & 0.18 & 77 & 1.21 & $0.5-0.6$ \\
LES Breuer (2000) (Case A1) & 1.24 & - & 0.204 & 96 & 1.40 & 0.57 \\
LES Breuer (2000) (Case B1) & 1.45 & - & 0.204 & 95 & 1.76 & 0.34 \\
DES Travin et al. (2000) & 1.08 & 0.29 & 0.21 & 77 & 1.04 & 1.1 \\
\hline
\end{tabular}

Comparing Case 1 and Case 2, it can be seen that the $2^{\text {nd }}$ order upwind scheme (used in Case 1) gives an excessive recirculation length and under-predicted values of $-C_{p b}, C_{D}$ and $C_{L, \text { rms }}$ as a consequence of significant numerical dissipation due to the upwind nature of the scheme. These results are improved with the Limited Linear scheme (used for all other cases). This is one of the total variation diminishing schemes, which combine the upwind and centred schemes to minimize diffusion and retain stability (Versteeg and Malalasekera 2007). With improved temporal resolution (Case 3), both $C_{D}$ and $C_{L, \text { rms }}$ are reduced, especially $C_{L, \text { rms }}$ for which a difference of $14 \%$ is observed. However, as the spatial resolutions are coarse in Case 2 and 3, it is better to draw conclusions about the temporal resolution based on an improved spatial resolution, which is investigated further for Case 7 below. From Case 2 to Case 5, a shortened recirculation length is predicted, thus giving higher $C_{D}$ and $C_{L, \text { rms. }}$. The results from the finer grid of Case 5 give a better agreement with the experiments. However, for Case 6, although the finest grid and time step are used, the results move away from the experimental ones. It should be noted that for Case 6, early activation of the LES mode inside the boundary layer was found (not presented here) even though it is not common for the DDES model, which may be a reason for the unexpected prediction of all parameters.

The mesh which gives the best agreement with the experimental data is Case 7 in which the wake region of Case 5 is further refined. Both $C_{D}$ and $C_{L, \text { rms }}$ reduce slightly when the non-dimensional time step is decreased from 0.009 to 0.0045 , in Case 8, but the differences are smaller than those between Cases 2 and 3. In comparison to the mean values, the rms values are much more sensitive to the numerical resolution. Finally, improving the spanwise resolution on the basis of Case 7 leads to only a $1.6 \%$ increase in $C_{D}$ and a $4 \%$ change in $C_{L, \mathrm{rms}}$, as can be seen in Case 9.

More detailed comparisons in terms of the mean surface quantities and the wake region statistics are given in Figures 2 and 3. Figure 2(a) shows the the distribution of the mean pressure coefficient around the cylinder, while the skin friction along the cylinder surface, scaled by $\sqrt{R e}$, is given in Figure 2(b). The skin friction coefficient, $C_{f}$, is defined as $C_{f}=\frac{\tau_{w}}{0.5 \rho U_{\infty}^{2}}$, with the wall shear stress, $\tau_{w}=\mu\left(\frac{\partial u}{\partial y}\right)_{y=0}$, where $\mu$ is the dynamic viscosity, $u$ is the flow velocity parallel to the wall and $y$ is the distance normal to the wall. $\left(\frac{\partial u}{\partial y}\right)_{y=0}=0$ represents the separation point. Hence, the point where $C_{f}$ first crosses the $x$-axis is the separation point, and where it returns to positive values marks flow reattachment.

Figure 2(a) shows that as the grid is refined from Case 2 to Case 7 , the base pressure coefficient (i.e. for $\theta=180^{\circ}$ ) becomes closer to the measurement data. The base pressure can be an indicator for the behaviour of $C_{D}$, which has been found to agree well with the measurement for Case 7 (see Table 2). Furthermore, the minimum pressures from the current simulations are all slightly lower than that from the measurement, but higher than the LES results of Kim (2006). With refinements in the grid, the minimum pressure departs further away from the measurement. As mentioned above, grid refinements do not always lead to better agreement. In addition, the location of the minimum pressure occurs further downstream for the current simulations, which may account 

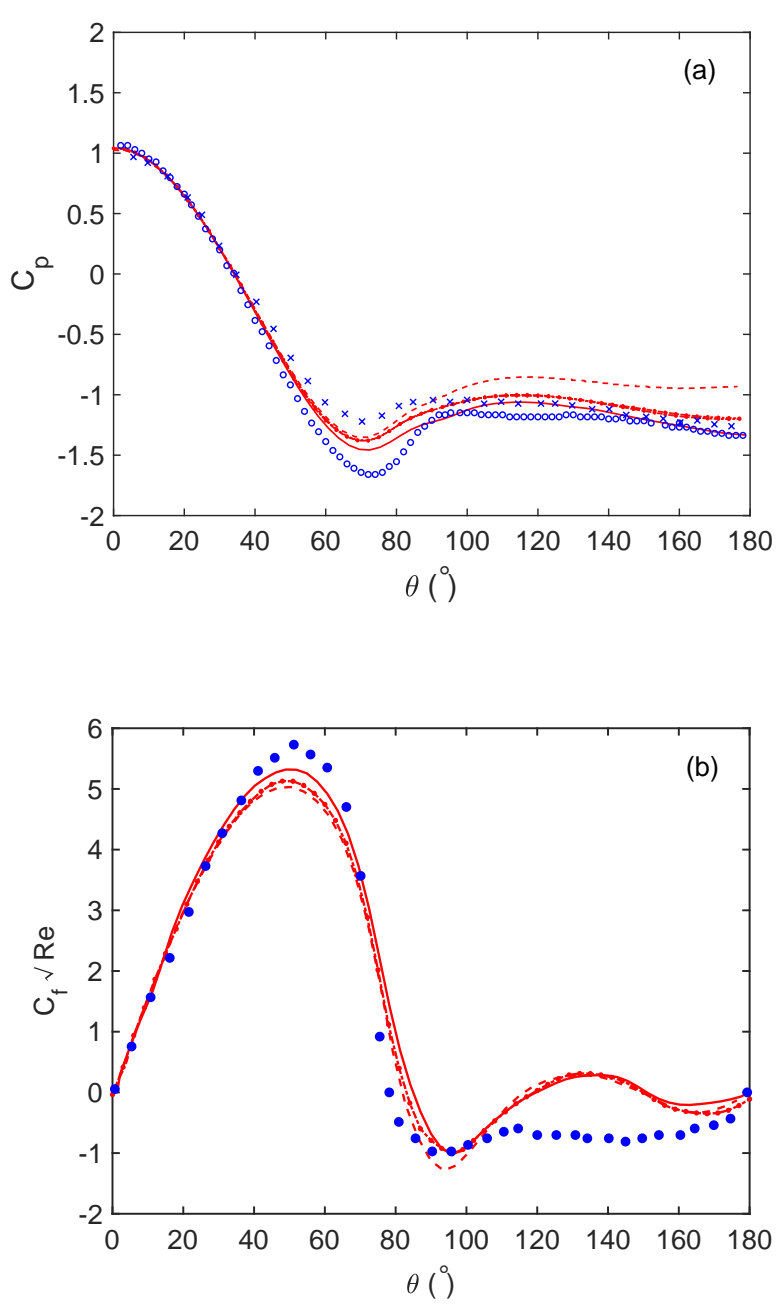

Figure 2. (a) Mean pressure coefficient along the cylinder surface. $\times$, Exp. $R e=1.4 \times 10^{5}$ (Cantwell and Coles 1983); o, LES. $R e=1.4 \times 10^{5}$ (Kim 2006). (b) Mean skin-friction coefficient scaled by $\sqrt{R e}$. Exp. Re $=1 \times 10^{5}$ (Achenbach 1968). --, Case 2; -.-, Case 5; -, Case 7.

for the delayed flow separations, as shown in Figure 2(b). A separation angle of $\theta=84^{\circ}$ is predicted in Case 7 which is higher than the measured value of $77^{\circ}$. The maximum of $C_{f}$ from Case 7 is lower than the experiment, with a difference of $6 \%$. At the rear of the cylinder, $C_{f}$ crosses the $x$-axis again and a region between $\theta=120^{\circ}-147^{\circ}$ exists with small positive $C_{f}$ in the current simulation although not in the experiment. This represents a small counter-rotating vortex due to the reattachment of the backflow. This positive $C_{f}$ region was also predicted by Travin et al. (2000) but in a slightly different range, $\theta=115^{\circ}-140^{\circ}$.

Figure 3(a) shows the profile of the mean streamwise velocity, $U_{x}$, along the centreline in the wake of the cylinder. Figure 3 (b) shows the normalized Reynolds stress, $\overline{u^{\prime} v^{\prime}} / U_{\infty}^{2}$, at $x / D=1$ which is $0.5 D$ away from the back of the cylinder. It can be seen in Figure 3(a) that refining the grid leads to a reduced recirculation length. The finest grid, Case 7, shows excellent agreement with the LES A1 results of Breuer (2000) for $x / D \leq 2.5$. For $x / D>2.5$, the LES results are generally lower than both the experiment and the current simulations. Although the minimum value of
$U_{x}$ and the recirculation length from Case 7 are slightly overpredicted compared with the experiment, the differences are small. A correct prediction for the recirculation length is important as it can reflect the primary vortex shedding process. It has been seen that a smaller $L_{r}$ can lead to increases in both $C_{D}$ and $C_{L \text {,rms. Normally, the Reynolds }}$ stresses in the wake region are not easy to predict. Nevertheless, a good agreement is obtained between Case 7 and the measurement, as shown in Figure 3(b), although the magnitudes of the peaks are slightly overpredicted. Notable differences with the measurement, e.g. the shifted peak location and the inflections between the peaks, are found for Case 2 and 5 and can be attributed to the overprediction of the recirculation length.
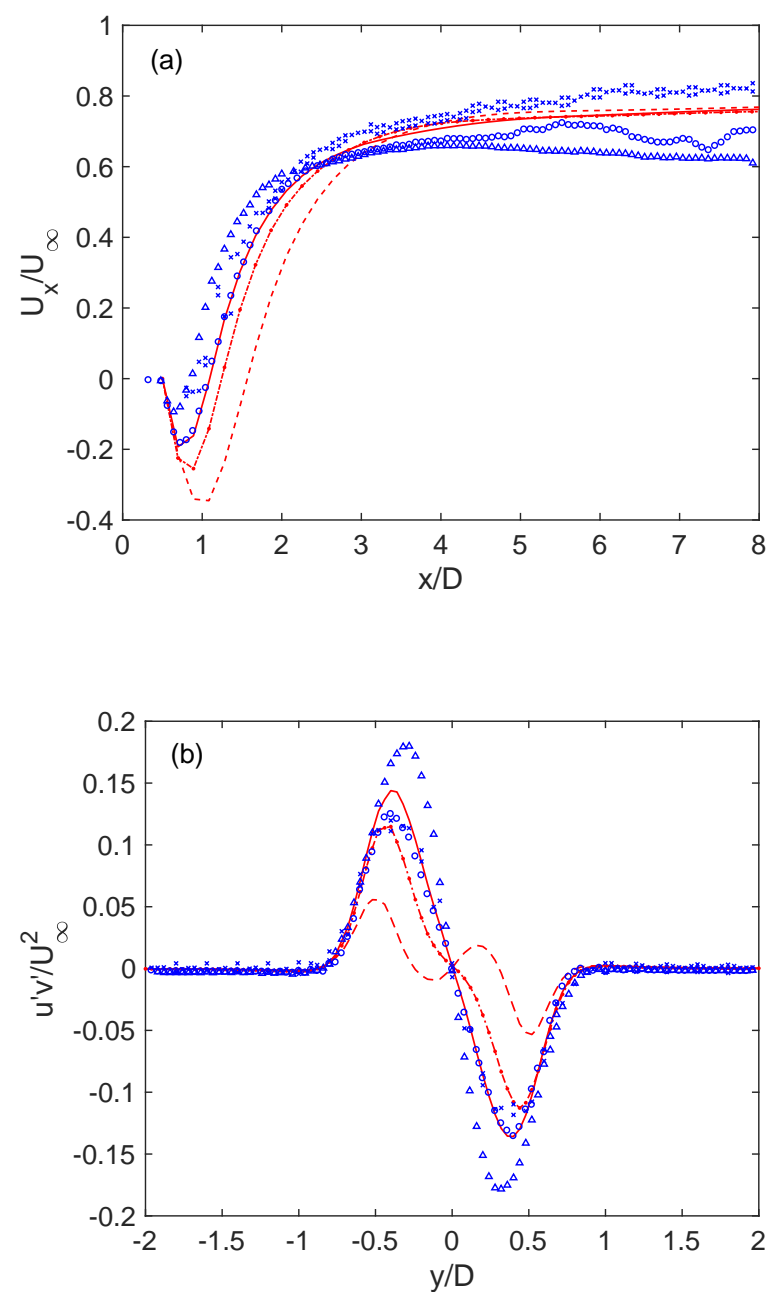

Figure 3. (a) Mean streamwise velocity along the centreline. (b) Mean Reynolds shear stresses at $x / D=1 . \times$, Exp. $R e=1.4 \times 10^{5}$ (Cantwell and Coles 1983); o, LES A1.

$R e=1.4 \times 10^{5}$ (Breuer 2000); $\triangle$, LES B1. Re $=1.4 \times 10^{5}$ (Breuer 2000);--, Case 2;---, Case 5; -, Case 7.

These comparisons suggest that DDES can provide fairly reliable predictions of the time-averaged and rms quantities. Since Case 7 gives the best agreement with both experimental and previous LES results in terms of $C_{D}$, $S t_{p}$ and $C_{L, \mathrm{rms}}$, it is therefore used for $R e=1 \times 10^{5}$ in the remainder of the study, see Table 1 . For other speeds the meshes are generated according to the same principles 
to provide similar resolution as Case 7. For example, the maximum cell aspect ratios in the first boundary layer remain nearly unchanged.

\section{Reynolds number effect}

Having established a suitable meshing strategy, this section studies the effect of varying the Reynolds number on aerodynamic and aeroacoustic characteristics of flow over a cylinder.

\section{Aerodynamic characteristics}

The results obtained for different Reynolds numbers are plotted in Figure 4 in terms of the drag coefficient, the peak Strouhal number and the rms value of lift coefficient. Available experimental and LES data from various sources are plotted for comparison. From the present simulations it can be seen that for $R e \leq 1 \times 10^{5}, C_{D}, S t_{p}$ and $C_{L, \mathrm{rms}}$ are more or less independent of $\operatorname{Re}$ with $C_{D}=1.0-1.2, S t_{p}=$ $0.19-0.20$ and $C_{L, \mathrm{rms}}=0.6-0.7$. These values show commendable agreement with the measurements.

As the Reynolds number is increased above $1 \times 10^{5}$, a sharp decrease occurs in $C_{D}$. This is often called the drag crisis, which marks the onset of the critical flow state. The Reynolds number here is called the critical Reynolds number which can vary significantly with different test conditions, such as different inflow turbulence levels or surface roughness (Norberg and Sunden 1987; Fage and Warsap 1930; Achenbach and Heinecke 1981). This critical Reynolds number is also extremely sensitive to numerical techniques (Rodríguez et al. 2015; Cheng et al. 2017). A small numerical perturbation introduced because of grid quality, numerical scheme or numerical model may be sufficient to trip the flow transition, which leads to a considerable scatter in the results in the literature. Nevertheless, on the whole, the value predicted by the current simulations, $R e=1 \times 10^{5}$, is within the acceptable range of the measurements and the LES simulations, and the trends in $C_{D}$ and $C_{L, \text { rms }}$ in the critical range are well predicted by the current simulations. As can be seen, shape decrease from 1.25 at $R e=1 \times 10^{5}$ to 0.54 at $R e=3.67 \times 10^{5}$ occurs for $C_{D}$, which is consistent with the measured data. $C_{L, \text { rms }}$ shows a similar trend, falling from 0.73 to a minimum of 0.058. The information in the literature on $C_{L, \mathrm{rms}}$ is rather limited for the critical range, and a large scatter exists. The rms value is normally difficult to predict, but DDES obtains a reasonable agreement with the measured data. As $C_{L, \mathrm{rms}}$ is an integration of the surface pressure fluctuations associated with the lift dipole, it is expected that the noise level will be related to this and will reduce greatly in the critical flow regime.

The significant changes in $C_{D}$ and $C_{L, \mathrm{rms}}$ in the critical flow regime can be associated with the length of the eddy formation region, $L_{r}$, which is defined as the distance from the back of the cylinder to the zero-mean-velocity point on the centreline. The variation in $L_{r} / D$ with $R e$ is plotted in Figure 5 which also includes the variations in the minimum pressure $\left(-C_{p \min }\right)$, the separation position $\left(\theta_{\text {sep }}\right)$ and base pressure $\left(-C_{p b}\right)$. The separation angle results should be treated with some care as the separation angle is sensitive to the numerical resolution, as shown in Section 3.
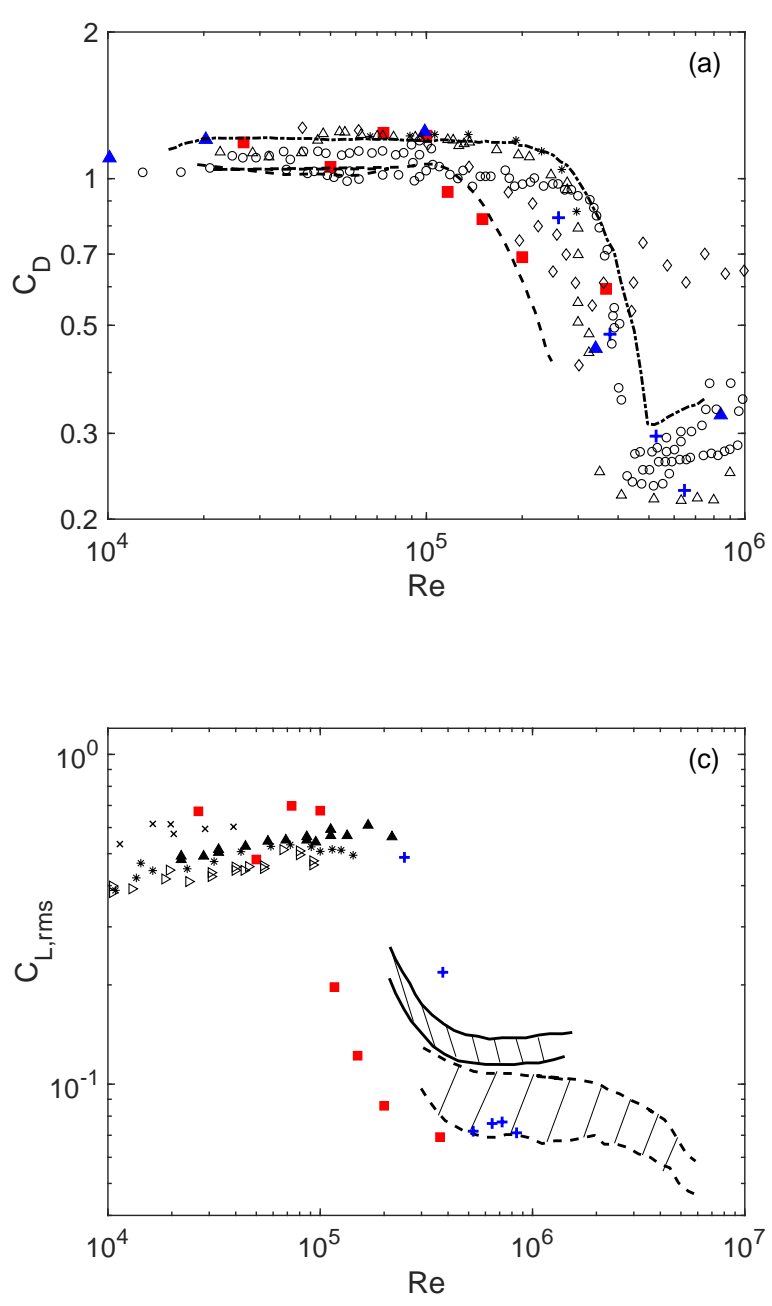

Figure 4. Variations of $C_{D}$ (a) and $C_{L \text {,rms }}$ (b) with Reynolds number. $\square$, present simulations; +, LES simulations of Rodríguez et al. (2015); $\mathbf{\Delta}$, LES simulations of Cheng et al. (2017). (a) $\diamond$, Achenbach (1968); ॰, Delany and Sorensen (1953); $\triangle$, Schewe (1983); *, Cantwell and Coles (1983); -.-, Wieselsberger (1922); - -, Fage and Warsap (1930). (b) $\times$, Moeller and Leehey (1984); $\triangleright$, Keefe (1962); *, Szepessy and Bearman (1993); $\boldsymbol{\Lambda}$, West and Apelt (1997); - Fung (2012); -, Loiseau and Szechenyi (1974).

Nevertheless, the same criteria for mesh generation are used at different speeds, which allows comparison between the cases. The resolutions of $\theta$ due to the mesh size around the cylinder, $\Delta \theta$, for different speeds are listed in Table 4 .

From Figure 5(a) and 5(b), it can be seen that $-C_{p \min }$ and $\theta_{\text {sep }}$ vary in a similar manner. This is due to the fact that flow separation is closely related to the region of favourable pressure gradient $(\partial P / \partial x>0)$ in the front part of the cylinder which enables the flow to accelerate. An increased favourable pressure gradient region, and thus a lower minimum pressure $\left(C_{p \min }\right)$, will lead to a delayed flow separation. For $R e \leq 10^{5},-C_{p b}$ and $\theta_{\text {sep }}$ remain almost constant, whereas for $R e>1.17 \times 10^{5},-C_{p \min }$ increases rapidly, which results in a larger region of favourable pressure gradient, and thus a delayed flow separation. High correlation is also found between $-C_{p b}$ and $L_{r}$. When the flow enters the critical state, the eddy formation length is 
Table 4. Resolutions of $\theta$ in mesh around cylinder.

\begin{tabular}{ccccccccc}
\hline$U_{\infty}[\mathrm{m} / \mathrm{s}]$ & 8 & 15 & 22 & 30 & 35 & 45 & 60 & 110 \\
$\Delta \theta\left[{ }^{\circ}\right]$ & 8.0 & 4.9 & 3.7 & 2.9 & 2.9 & 2.6 & 2.1 & 1.1 \\
\hline
\end{tabular}
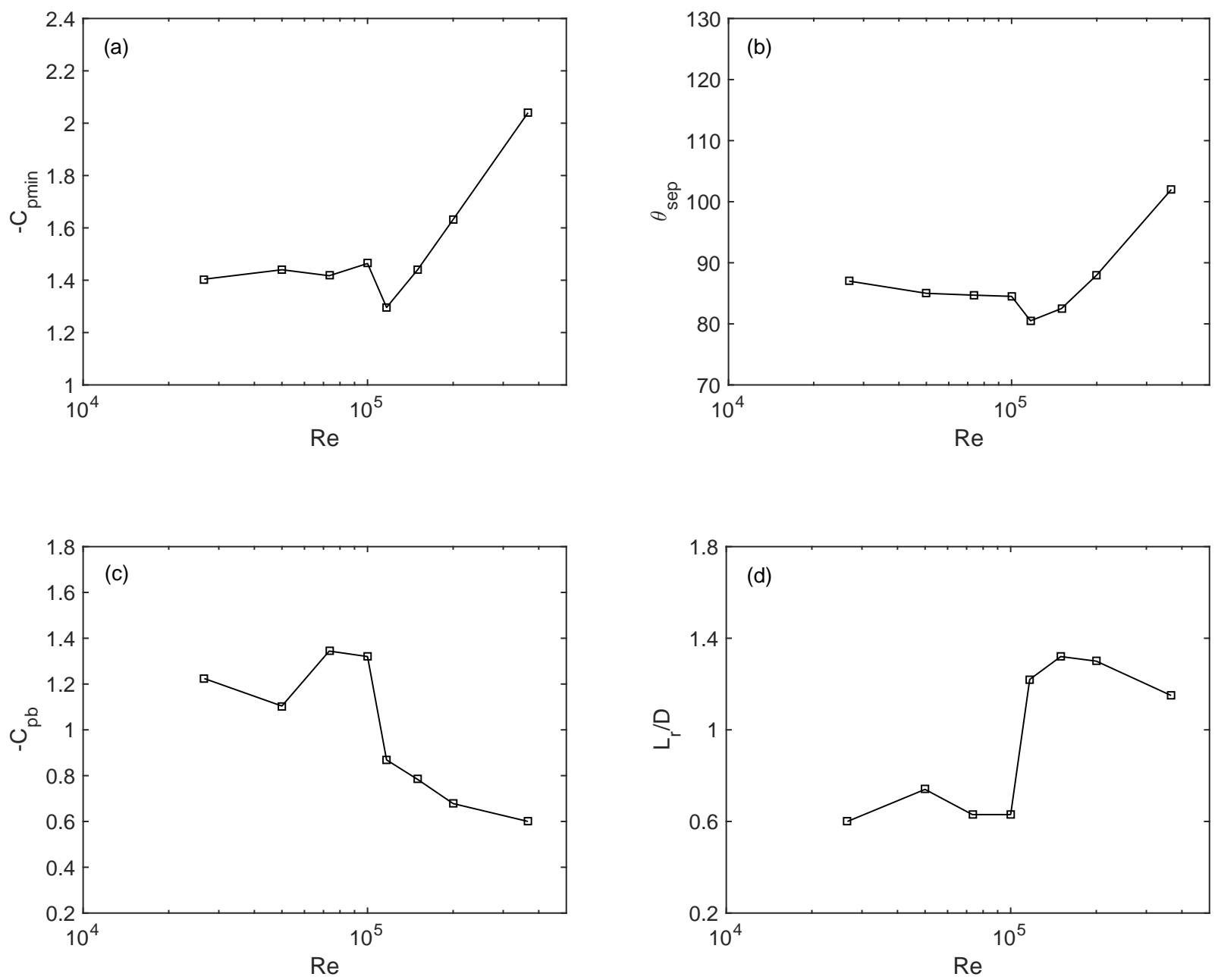

Figure 5. Variations of (a) $-C_{p m i n}$, (b) $\theta_{s e p}$, (c) $-C_{p b}$ and (d) $L_{r} / D$ with Reynolds number.

significantly extended and the low pressure region moves downstream away from the base of the cylinder. This results in a decrease in $-C_{p b}$ and weakens the alternate pressure fluctuations on the cylinder surface; therefore $C_{L, \text { rms }}$ reduces as well.

\section{Aeroacoustic results}

Time histories of flow solutions from CFD are fed to a FW$\mathrm{H}$ solver for far-field noise prediction, with the integration surface placed on the cylinder. 72 far-field receivers are uniformly distributed on a plane parallel to the incoming flow with a radius $R=100 D=5 \mathrm{~m}$ from the cylinder surface. $\theta=90^{\circ}(\theta$ is measured clockwise from the leading edge of the cylinder) is directly above the cylinder. The predicted farfield pressure time history is used to calculate its spectrum. The power spectral density (PSD) is computed based on Welch's method using a Hanning window with an overlap of $50 \%$ following Hu et al. (2006).

Figure 6 compares the 1/6 octave band noise spectra from the current simulations at the Reynolds numbers in the subcritical range $\left(R e=2.67 \times 10^{4}-1 \times 10^{5}\right)$ with the measurement of Latorre Iglesias et al. (2016). The measurement was carried out in an open jet anechoic wind tunnel for $R e=2.59 \times 10^{4}$ (subcritical). The receiver is located at $\theta=90^{\circ}$ where the radiated noise is mainly influenced by the lift fluctuations. In the simulations, the surface pressure, used as input for the noise calculation, was sampled over 60 shedding cycles after the flow had statistically converged. The measurement data were scaled by $U_{\infty}$ (to the speed of $30 \mathrm{~m} / \mathrm{s}$ ), $D, L$, and $r$ based on Equation (1) to allow comparison with the simulated results. The simulations at different speeds are also normalized to $U_{\infty}=30 \mathrm{~m} / \mathrm{s}$. The narrow band spectra were then converted to $1 / 6$ octave bands, which are chosen to provide sufficient details of the spectral shape while allowing the spectra to be easily distinguishable. Finally the results are plotted against Strouhal number, St.

As shown in Figure 6, the spectra are characterised by a dominant peak which is associated with the fundamental vortex shedding. The normalized frequency of this primary 


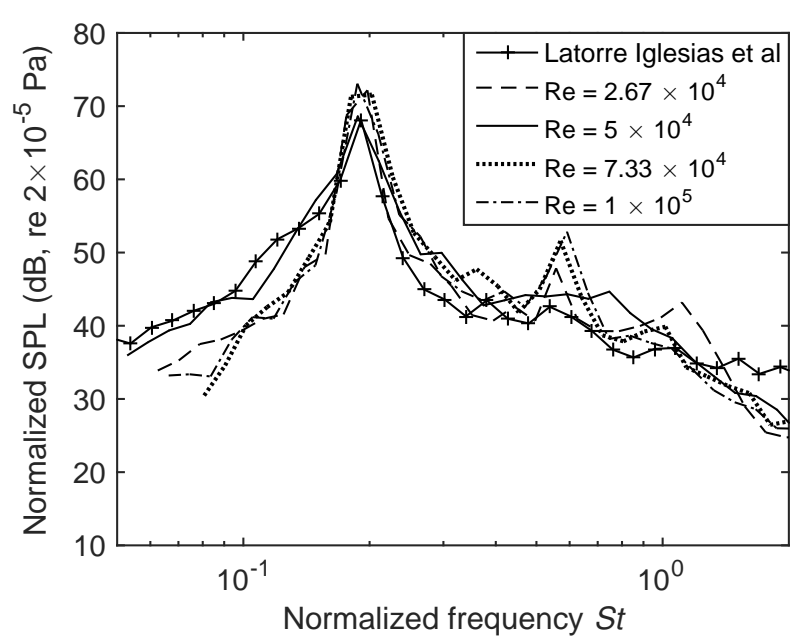

Figure 6. Comparison of the normalized $1 / 6$ octave band noise spectra between current simulations and the measurements by Latorre Iglesias et al $\left(R e=2.59 \times 10^{4}\right)$.

peak obtained from the simulations varies slightly with the Reynolds number and deviates slightly from the measurement. However, the deviations are caused by the different central frequencies of the 1/6 octave bands for these cases when expressed in terms of St. From the narrow band spectra (not shown here), $S t_{p}=0.183$ is found for the measurement and values in the range $0.19-0.20$ for the simulations.

Good agreement is found for the level of the primary peak between the simulation at $R e=5 \times 10^{4}$ and the measurement. Compared with other Reynolds numbers, the spectrum at $R e=5 \times 10^{4}$ has a broader primary peak with a slightly lower noise level. This implies that the flow at this Reynolds number is less coherent in vortex shedding and more three-dimensional, which has been confirmed by investigation of the correlation coefficient (see Section 5 below). The deviation in the normalized spectral peak level between different Reynolds numbers is up to $4.5 \mathrm{~dB}$. In addition to the primary peak, a harmonic peak at three times the frequency of the primary peak is found for both the measurement and the simulations at three of the speeds, but the peak has higher levels in the simulations. Differences with the measurement are also observed at frequencies below the primary peak. The predicted levels are generally lower except at $R e=5 \times 10^{4}$. It is worth pointing out that in the measurement, free ends of the cylinder were used which could cause some addition noise that can influence the overall spectrum.

In order to assess the effect of the Reynolds number on the noise in the critical flow regime, the 1/6 octave band noise spectra for $R e \geq 1 \times 10^{5}$ are presented in Figure 7 . For easy comparison the noise level has been corrected to that at $R e=1 \times 10^{5}$ based on the speed factor of $U^{6}$ in Equation (1). As can be seen, the frequency of the primary peak gradually increases at higher Reynolds numbers and no second harmonic peak is found at these Reynolds numbers in the critical regime. The increase in $S t_{p}$ in the critical region was also pointed out by Fujita (2010) who carried out measurements in a low-noise wind tunnel with end plates applied. In his measurements, the value of $S t_{p}$ was found to increase from around 0.2 in the subcritical range to a maximum of 0.45 at $7.4 \times 10^{5}$. Figure 7 shows a significant drop in level occurs between $R e=1 \times 10^{5}$ and $1.17 \times 10^{5}$ at the primary peak and at high frequencies, but the drop is not as large at low frequencies. Above $R e=1.17 \times 10^{5}$, the noise level still decreases with increasing $R e$ but to a smaller extent. The maximum difference in the spectral peak level between the various cases is about $17 \mathrm{~dB}$, which indicates the great effect of the Reynolds number on the noise level in the critical flow regime when the velocity factor $\left(U^{6}\right)$ is taken out.

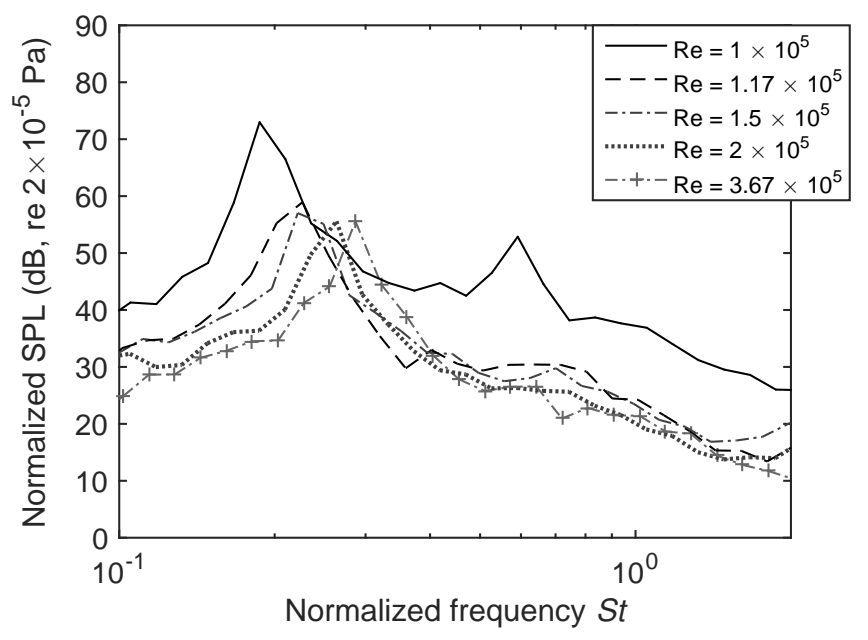

Figure 7. Normalized $1 / 6$ octave band noise spectrum for different Reynolds numbers in the critical flow regime.

The variation in the peak level of the 1/6 octave band spectra and the overall sound pressure level (OASPL) with Reynolds number is presented in Figure 8. Here the OASPL is determined over the normalized frequency range $S t=$ $0.05-2$. The level difference $\Delta \mathrm{SPL}$ is defined here as,

$$
\Delta \mathrm{SPL}=\mathrm{SPL}_{U}-60 \log _{10}(U)-\mathrm{SPL}_{\text {average }}
$$

where $S P L_{\text {average }}$ is obtained by averaging the noise levels for subcritical Reynolds numbers, after removing the speed dependence, by subtracting a factor of $60 \log _{10} U . \Delta$ SPL is only expected to be related to $S t_{p}, C_{L, \text { rms }}$ and $l_{c}$, which are dependent on the Reynolds number. As can be seen, similar trends are obtained for the $1 / 6$ octave peak SPL and the OASPL as a function of $R e$ and the reductions in them in the critical Reynolds number range are nearly equivalent. In Figure 8, the measured results from Fujita (2010) are also presented. In these measurements, the onset of the critical regime is at $R e=3 \times 10^{5}$. To allow for comparison, the results are plotted against $R e / R e_{\text {crit }}$. The results for $\Delta$ SPL show a similar behaviour to the plot of $C_{L, \text { rms }}$ against the Reynolds number (Figure 4(b)). The noise levels are found to vary within $3 \mathrm{~dB}$ in the subcritical Reynolds number range, while in the critical range, a significant Reynolds number effect is found. A sharp decrease of $12 \mathrm{~dB}$ occurs immediately above the critical Reynolds number $1 \times 10^{5}$. After this, $\triangle$ SPL decreases more slowly and the level is almost unchanged from $R e=2 \times 10^{5}$ to $R e=3.67 \times 10^{5}$. The maximum decrease in the critical regime is about 17.5 $\mathrm{dB}$ in the present simulations. This shows a good agreement with the change in the noise level of $18.5 \mathrm{~dB}$ in the 
measurements although the measured result appears to level off more quickly.

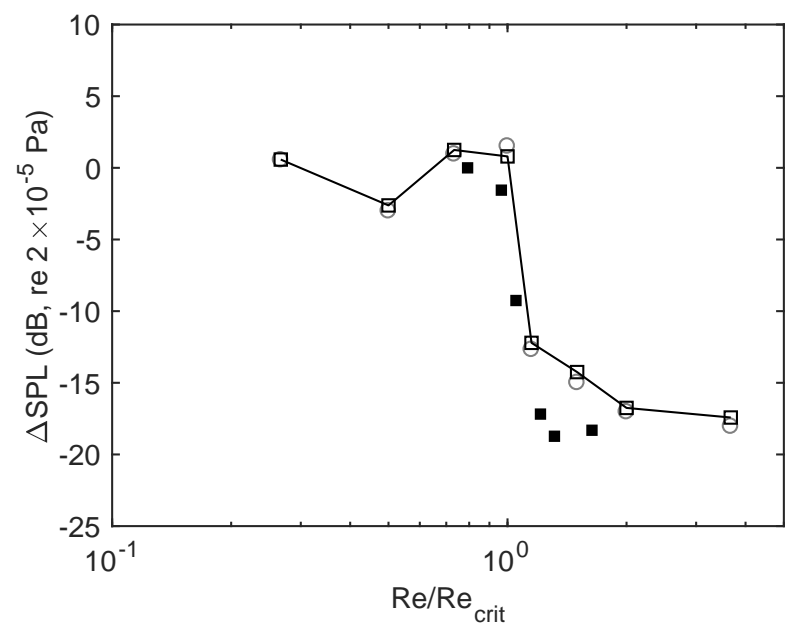

Figure 8. Variations of $\Delta S P L$ with Reynolds number. $\circ, 1 / 6$ octave peak SPLs from present simulations; $\square$, from present simulations $R e_{\text {crit }}=1 \times 10^{5} ; \mathbf{\square}$, from experiments of Fujita (2010) $R e_{\text {crit }}=3 \times 10^{5}$.

Figure 9 shows the far-field noise directivity for different Reynolds numbers. Again the flow speed effect has been subtracted. As can be seen, a dipole noise pattern is predicted for all the presented Reynolds numbers with the maximum located at $\theta=90^{\circ}, 270^{\circ}$, which is mostly influenced by the lift fluctuation, and the minimum at $\theta=0^{\circ}, 180^{\circ}$, determined by the drag fluctuation. The extent of the noise reduction in the critical range is similar for the lift and drag components. The differences between the maxima and the minima are generally around $16 \mathrm{~dB}$ in the subcritical Reynolds number range except at $R e=1 \times 10^{5}$, where a much lower noise level appears at $\theta=0^{\circ}$ and $180^{\circ}$, resulting in a higher difference of about $26 \mathrm{~dB}$. In the critical Reynolds number range a difference of about $18 \mathrm{~dB}$ is found, which is similar to that found in the subcritical range.

\section{Effect of the spanwise domain length}

As discussed in Section 2.2.1, in the current simulations, a spanwise length of $3 D$ has been used for the simulations at Reynolds numbers, $R e=2.67 \times 10^{4}-3.67 \times 10^{5}$. In this section the effect of the spanwise domain size on noise results is investigated. In order to clarify the extent to which the spanwise flow features are captured by the simulations, correlations along the spanwise direction for all Reynolds numbers are calculated and presented in Figure 10. The correlation coefficient for pressure is given as,

$$
R_{a b}=\frac{\overline{p_{a} p_{b}}}{p_{\mathrm{rms}, a} p_{\mathrm{rms}, b}}
$$

Here $\overline{p_{a} p_{b}}$ indicates the product of fluctuating pressure at two points a and b separated by $\Delta z$ along the span, which is averaged over the span and time. Since periodic boundary conditions are employed in the computations, the maximum separation that can be considered is half of the span length.

Figures 10(a) and 10(b) show the results for the surface points at $\theta=90^{\circ}$, which is close to the mean flow separation

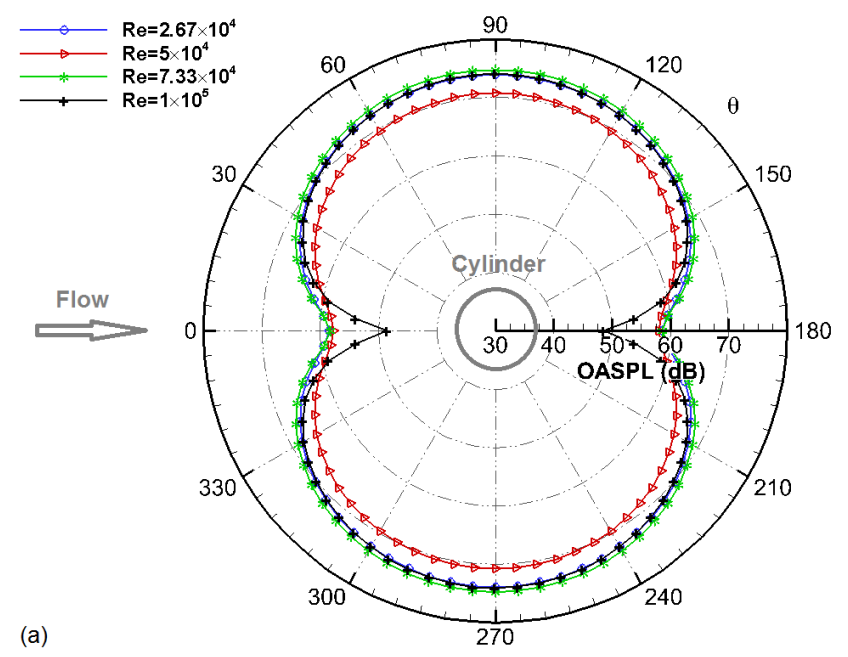

(b)

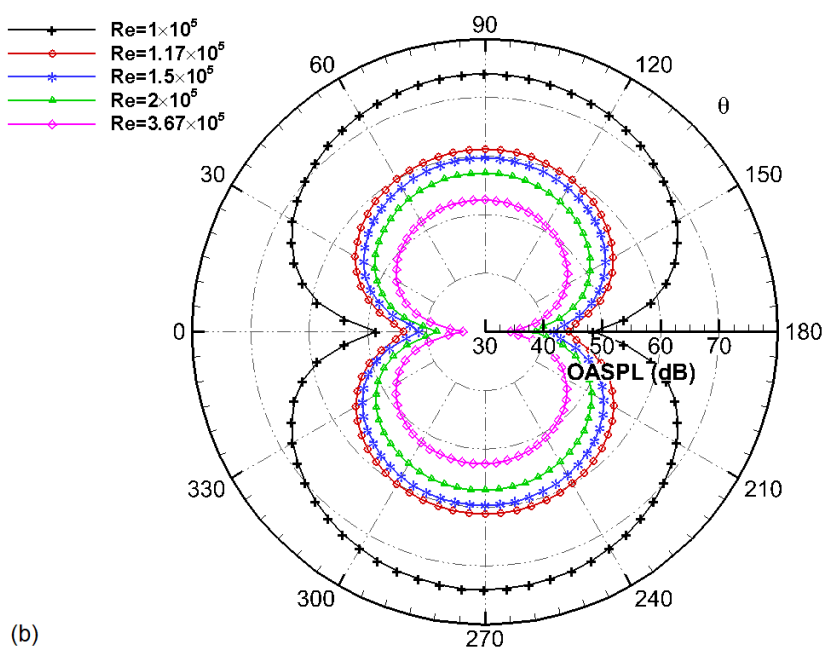

Figure 9. Far-field noise directivity for different Reynolds number normalised to $30 \mathrm{~m} / \mathrm{s}\left(\operatorname{Re}=1 \times 10^{5}\right)$. (a) Subcritical flow regime; (b) critical flow regime.

point $\left(\theta=85^{\circ}-90^{\circ}\right)$, while Figures $10(\mathrm{c})$ and $10(\mathrm{~d})$ are at $\theta=120^{\circ}$ which is behind the separation point. As can be seen from Figure 10(a), for subcritical Reynolds numbers, the correlation coefficients at $\theta=90^{\circ}$ hardly drop with increasing separation distance; there is an exception at $R e=$ $5 \times 10^{4}$, where a relatively low value of 0.45 is found at the maximum separation distance. A lower correlation coefficient indicates enhanced three-dimensionality of the flow. This explains the lower and broader peak that was found at $R e=5 \times 10^{4}$ for the noise spectrum (see Figure 6). As $R e$ is increased to the critical range, the surface pressure becomes much less correlated, which indicates a more threedimensional flow. Figure 10(b) shows that the correlation coefficient at the maximum separation distance reduces from around 0.8 in the subcritical range to 0.4 at $R e=1.5 \times 10^{5}$, and thereafter to less than 0.2 at $R e=2 \times 10^{5}$ and $3.65 \times$ $10^{5}$. Smaller correlation lengths are therefore expected for these critical Reynolds numbers.

The correlation coefficients decrease more rapidly at $\theta=$ $120^{\circ}$ for both subcritical and critical Reynolds numbers, as can be seen in Figure 10(c) and 10(d). This is due 

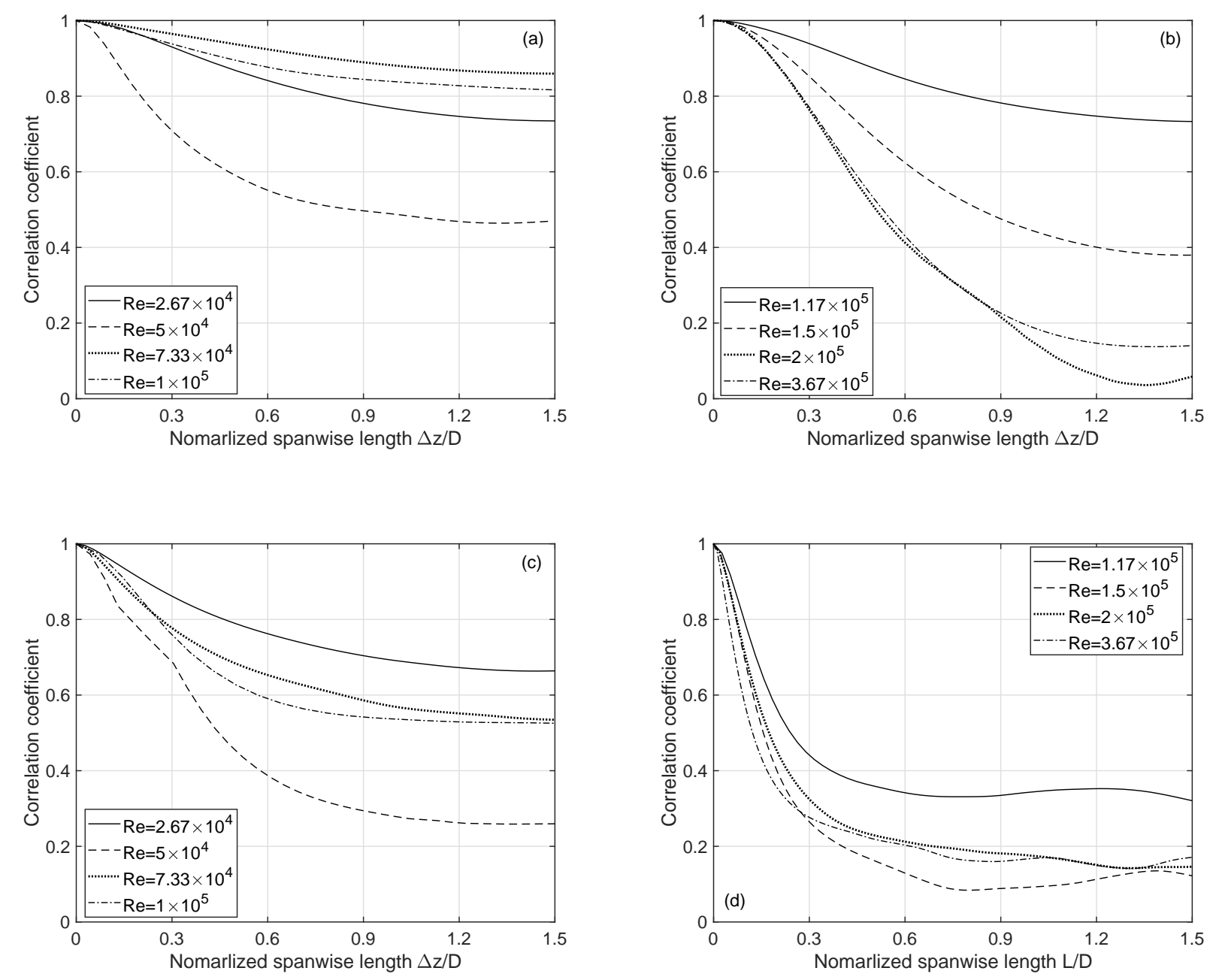

Figure 10. Correlation coefficient as a function of separation distance for different Reynolds numbers at different surface locations. (a) $\theta=90^{\circ}$, subcritical $R e$; (b) $\theta=90^{\circ}$, critical $R e$; (c) $\theta=120^{\circ}$, subcritical $R e$; (d) $\theta=120^{\circ}$, critical $R e$.

to the fact that the spanwise structures are well-correlated before or just after the flow separation, e.g. at $\theta=90^{\circ}$, whereas these structures break down into smaller scales as they develop, resulting in weaker coherence at $\theta=120^{\circ}$. Especially for Reynolds numbers in the critical range, the minimum correlation coefficients reduce to generally less than 0.2 at this location. This suggests that a span length of $3 D$ is sufficient to represent the flow features in the critical range.

Although it has been found by other researchers that there are only small differences in aerodynamic results with span lengths up to $2 \pi D$, and most work has used span lengths between $1 D$ and $\pi D$ (Breuer 2000; Travin et al. 2000; Kim 2006; Mockett et al. 2009), it is still necessary to clarify to what extent the noise level will be affected by changes in the spanwise length in the model. The spanwise correlation length is important for noise prediction as indicated in Equation (1), so the spanwise domain size has to be sufficient to include several correlated segments.

Additional simulations with longer spanwise dimensions are therefore carried out. It has been found from Figure 10 that the minimum correlation coefficient did not drop much for subcritical Reynolds numbers, but reduced to lower values for most of the Reynolds numbers in the critical regime. Therefore, the study here focuses mainly on subcritical Reynolds numbers. Figure 11 shows the variations in the correlation coefficient obtained with different spanwise lengths for different Reynolds numbers. The spanwise grid resolution is kept the same. The results are presented for $\theta=90^{\circ}$ where the coefficient drops slowest. As can be seen, for all these Reynolds numbers, the minimum correlation coefficient decreases gradually with increasing spanwise length. For $R e=2.67 \times 10^{4}$, a sufficiently low value below 0.2 at the maximum separation distance is obtained when the spanwise length is increased to $24 D$. A correlation length of $3.6 D$ was then calculated by integrating the coefficient over half of the spanwise length, which is consistent with the value of about $4 D$ indicated by Norberg (2003) at similar Reynolds numbers. When the Reynolds number is increased (see Figure 11(b) and 11(c)), the spanwise length required to make the correlation coefficient reduce to low values becomes shorter; this is $6 D$ for $R e=5 \times 10^{4}$ and $9 D$ for $1 \times 10^{5}$. In addition, negative values of correlation coefficient are found for $R e=1 \times$ $10^{5}$ with $L=18 D$, which may be due to the insufficient sampling time. The influence of the spanwise length on the 

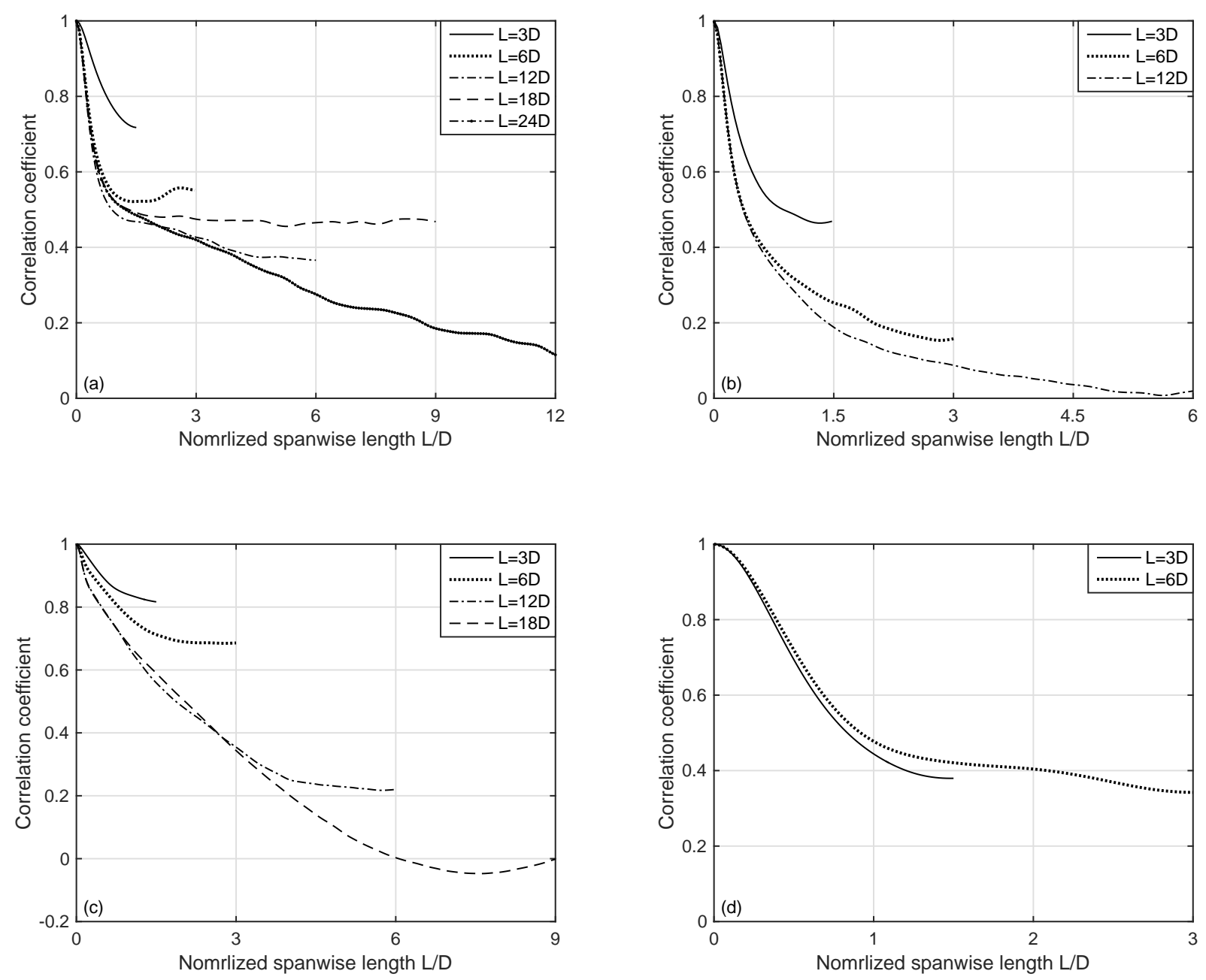

Figure 11. Correlation coefficients as a function of the spanwise separation distance for different spanwise lengths. (a) $U_{\infty}=8$ $\mathrm{m} / \mathrm{s}, R e=2.67 \times 10^{4}$; (b) $U_{\infty}=15 \mathrm{~m} / \mathrm{s}, R e=5 \times 10^{4}$; (b) $U_{\infty}=30 \mathrm{~m} / \mathrm{s}, R e=1 \times 10^{5}$; (b) $U_{\infty}=45 \mathrm{~m} / \mathrm{s}, R e=1.5 \times 10^{5}$.

correlation is also investigated for a Reynolds number in the critical region, $R e=1.5 \times 10^{5}$, as shown in Figure $11(\mathrm{~d})$. It seems that doubling the spanwise length to $6 D$ hardly changes the correlation in this case. Longer span lengths are not considered for this Reynolds number.

Far-field noise is calculated in all cases with different spanwise domain lengths. The peak Strouhal number is found to be insensitive to the spanwise dimension. However, the spectral level varies. Figure 12 shows the changes in the far-field noise level, represented by OASPL, caused by the spanwise length included in the model. $\triangle \mathrm{OASPL}$ is the difference in the level between different spanwise lengths and a reference length $L_{\text {ref }}=3 D$ after applying the length correction, $10 \log _{10}\left(L / L_{\text {ref }}\right)$. It is shown that for $R e=2.67 \times 10^{4}$, the maximum variation when changing the spanwise dimension is about $4.5 \mathrm{~dB}$ at $L=18 D$. When further increasing it to $L=24 D$, the level is nearly unchanged. For $R e=1 \times 10^{5}$, the maximum variation is 2.6 $\mathrm{dB}$ at $L=12 D$, but when the length is further increased to $18 D$, the level difference reduces to $1.5 \mathrm{~dB}$. As this corresponds to the critical Reynolds number, the rms lift coefficient may be affected when varying the spanwise length for this case. For $R e=5 \times 10^{4}$ and $1.5 \times 10^{5}$, shorter spanwise lengths are sufficient to obtain converged data, with variations of only $1.0 \mathrm{~dB}$ and $0.3 \mathrm{~dB}$ respectively when the span is extended beyond $L=3 D$.

In the current research, a large number of cases have been run. The computations would be unaffordable if a long spanwise length was used to achieve sufficiently low values of the correlation. Therefore the predictions have been based on a span length of $3 D$ while recognising that the sound level may be under-predicted by up to around $4.5 \mathrm{~dB}$ for Reynolds numbers in the subcritical regime. The variations for Reynolds numbers in the critical region appear to be at most $1.5 \mathrm{~dB}$.

\section{Conclusions}

The aerodynamic noise from circular cylinders at Reynolds numbers $2.67 \times 10^{4}-3.67 \times 10^{5}$ have been investigated by using a hybrid method of CFD analysis based on DDES and the FW-H acoustic analogy. A grid sensitivity study, carried out at $R e=1 \times 10^{5}$, showed a reasonable convergence although $C_{L, \mathrm{rms}}$ was sensitive to the grid refinement. In spite of the sensitivity, good agreement was obtained with the measurements for both mean and 


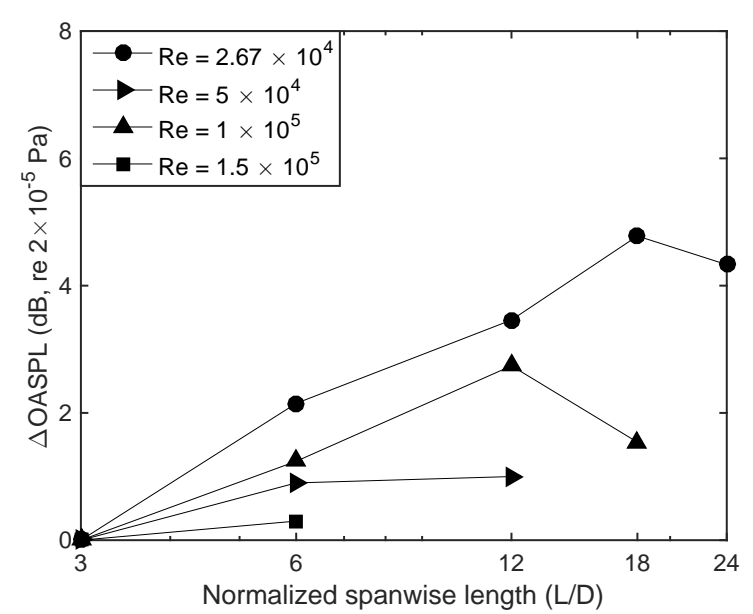

Figure 12. Variations in OASPLs obtained with different spanwise length.

fluctuating flow quantities. The predicted noise spectra also gave commendable agreement with measurements for the peak frequency and the peak level.

The effect of varying the Reynolds number on the aerodynamic and aeroacoustic characteristics was investigated. As a compromise between the required accuracy and affordable computational cost, DDES has been shown in the present study to be a practical tool that can provide reasonable predictions for the time-averaged and rms quantities. The results show a sharp decrease in $C_{D}$ and $C_{L, r m s}$ accompanied by an increase in $S t_{p}$ for the critical Reynolds numbers. These changes are comparable to values from measurements. The flow in the subcritical regime is found to be insensitive to the Reynolds number and only small variations exist in the noise level and the peak frequency after allowing for the speed effect. Significant reductions in the noise levels of up to $17 \mathrm{~dB}$ were found in the critical Reynolds number range, corresponding to $R e \geq 1.17 \times 10^{5}$ in the current simulations. The amount of reduction is close to that reported by Fujita (2010) of about $18.5 \mathrm{~dB}$. The reductions can be attributed to the much lengthened eddy formation region and the enhanced three-dimensionality that have been observed in the current simulations.

As the spanwise correlation length is important in predicting the radiated noise, a sufficient computational dimension is needed. A spanwise length of $3 D$ was found to be sufficient for most critical Reynolds numbers to allow the correlation coefficient reduce to low values, whereas for most subcritical Reynolds numbers, longer spanwise lengths were necessary. Using a spanwise length of $3 D$ the sound level may be under-predicted by up to about $4 \mathrm{~dB}$ for subcritical Reynolds numbers.

\section{Acknowledgements}

All simulations in this paper were run on Iridis 4 Super Computer at the University of Southampton. The authors are also grateful to Arup for financial support.

\section{References}

Achenbach E (1968) Distribution of local pressure and skin friction around a circular cylinder in cross-flow up to $R e=5 \times 10^{6}$.
Journal of Fluid Mechanics 34(4): 625-639.

Achenbach E and Heinecke E (1981) On vortex shedding from smooth and rough cylinders in the range of Reynolds numbers $6 \times 10^{3}$ to $5 \times 10^{6}$. Journal of Fluid Mechanics 109: 239-251.

Bearman P (1969) On vortex shedding from a circular cylinder in the critical Reynolds number regime. Journal of Fluid Mechanics 37(3): 577-585.

Breuer M (2000) A challenging test case for large eddy simulation: high Reynolds number circular cylinder flow. International Journal of Heat and Fluid Flow 21(5): 648-654.

Cantwell B and Coles D (1983) An experimental study of entrainment and transport in the turbulent near wake of a circular cylinder. Journal of Fluid Mechanics 136: 321-374.

Cheng W, Pullin D, Samtaney R, Zhang W and Gao W (2017) Large-eddy simulation of flow over a cylinder with $R e_{D}$ from $3.9 \times 10^{3}$ to $8.5 \times 10^{5}$ : a skin-friction perspective. Journal of Fluid Mechanics 820: 121-158.

Curle N (1955) The influence of solid boundaries upon aerodynamic sound. In: Proceedings of the Royal Society of London A: Mathematical, Physical and Engineering Sciences, volume 231. The Royal Society, pp. 505-514.

Delany NK and Sorensen NE (1953) Low-speed drag of cylinders of various shapes. Nat. Adv. Comm. Aero., Wash., Tech. Notes : 3038 .

Fage A and Warsap J (1930) The effects of turbulence and surface roughness on the drag of a circular cylinder. In: Technical Report R\&M 1283. Aeronautical Research Council.

Farell C and Blessmann J (1983) On critical flow around smooth circular cylinders. Journal of Fluid Mechanics 136: 375-391.

Fröhlich J and von Terzi D (2008) Hybrid LES/RANS methods for the simulation of turbulent flows. Progress in Aerospace Sciences 44(5): 349-377.

Fujita H (2010) The characteristics of the Aeolian tone radiated from two-dimensional cylinders. Fluid Dynamics Research 42(1): 015002.

Fung Y (2012) Fluctuating lift and drag acting on a cylinder in a flow at supercritical Reynolds numbers. Journal of the Aerospace Sciences 27(11): 801-814.

Hu Z, Morfey CL and Sandham ND (2006) Wall pressure and shear stress spectra from direct simulations of channel flow. AIAA Journal 44(7): 1541-1549.

Keefe RT (1962) Investigation of the fluctuating forces acting on a stationary circular cylinder in a subsonic stream and of the associated sound field. The Journal of the Acoustical Society of America 34(11): 1711-1714.

Kim SE (2006) Large eddy simulation of turbulent flow past a circular cylinder in subcritical regime. AIAA paper 1418: 912.

King W and Pfizenmaier E (2009) An experimental study of sound generated by flows around cylinders of different cross-section. Journal of Sound and Vibration 328(3): 318-337.

Latorre Iglesias E, Thompson D and Smith M (2016) Experimental study of the aerodynamic noise radiated by cylinders with different cross-sections and yaw angles. Journal of Sound and Vibration 361: 108-129.

Lockard DP, Khorrami MR, Choudhari MM, Hutcheson FV, Brooks TF and Stead DJ (2007) Tandem cylinder noise predictions. AIAA paper 3450: 2007. 
Loiseau H and Szechenyi E (1974) Dynamic lift on a cylinder in high Reynolds number flow. Flow Induced Structural Vibrations : 755-761.

Mockett C, Perrin R, Reimann T, Braza M and Thiele F (2009) Analysis of detached-eddy simulation for the flow around a circular cylinder with reference to piv data. In: IUTAM Symposium on Unsteady Separated Flows and their Control. Springer, pp. 417-427.

Moeller M and Leehey P (1984) Unsteady forces on a cylinder in cross flow at subcritical Reynolds numbers. In: ASME Symposium on Flow-induced Vibrations, volume 1. pp. 57-71.

Norberg C (2003) Fluctuating lift on a circular cylinder: review and new measurements. Journal of Fluids and Structures 17(1): 57-96.

Norberg C and Sunden B (1987) Turbulence and Reynolds number effects on the flow and fluid forces on a single cylinder in cross flow. Journal of Fluids and Structures 1(3): 337-357.

Rodríguez I, Lehmkuhl O, Chiva J, Borrell R and Oliva A (2015) On the flow past a circular cylinder from critical to super-critical Reynolds numbers: Wake topology and vortex shedding. International Journal of Heat and Fluid Flow 55: 91-103.

Roshko A (1961) Experiments on the flow past a circular cylinder at very high Reynolds number. Journal of Fluid Mechanics 10(3): 345-356.

Schewe G (1983) On the force fluctuations acting on a circular cylinder in crossflow from subcritical up to transcritical Reynolds numbers. Journal of Fluid Mechanics 133: 265-285.

Spalart PR (2000) Strategies for turbulence modelling and simulations. International Journal of Heat and Fluid Flow 21: 252-263.

Spalart PR, Deck S, Shur ML, Squires KD, Strelets MK and Travin A (2006) A new version of detached-eddy simulation, resistant to ambiguous grid densities. Theoretical and Computational Fluid Dynamics 20(3): 181.

Szepessy S and Bearman P (1993) Analysis of a pressure averaging device for measuring aerodynamic forces on a circular cylinder. Experiments in Fluids 16(2): 120-128.

Thompson DJ, Latorre Iglesias E, Liu X, Zhu J and $\mathrm{Hu} \mathrm{Z}$ (2015) Recent developments in the prediction and control of aerodynamic noise from high-speed trains. International Journal of Rail Transportation 3(3): 119-150.

Travin A, Shur M, Strelets M and Spalart P (2000) Detached-eddy simulations past a circular cylinder. Flow, Turbulence and Combustion 63(1): 293-313.

Versteeg HK and Malalasekera W (2007) An introduction to computational fluid dynamics: the finite volume method. Pearson Education.

West G and Apelt C (1997) Fluctuating lift and drag forces on finite lengths of a circular cylinder in the subcritical Reynolds number range. Journal of Fluids and Structures 11(2): 135158.

Wieselsberger C (1922) New data on the laws of fluid resistance. Tech. Rep. NACA TN-84 NACA .

Williams JF and Hawkings DL (1969) Sound generation by turbulence and surfaces in arbitrary motion. Philosophical Transactions of the Royal Society of London A: Mathematical, Physical and Engineering Sciences 264(1151): 321-342.
Zdravkovich M (1997) Flow Around Circular Cylinders; vol. 1. Fundamentals (two volumes). Oxford University Press. 